\title{
Tumor lysis syndrome associated with chemotherapy in primary retroperitoneal soft tissue sarcoma by ex vivo ATP-based tumor chemo-sensitivity assay (ATP-TCA)
}

\author{
Ke-Qing Qian' \\ Heng Ye' \\ Yi-Wen Xiao' \\ Yong-Yi Bao \\ Chun-Jian $\mathrm{Qi}^{1}$ \\ 'Department of Oncology; \\ ${ }^{2}$ Department of Pathology, the \\ Changzhou No. 2 People's Hospital \\ Affiliated to Nanjing Medical \\ University, Changzhou, China
}

Correspondence: Ke-Qing Qian Department of Oncology, The Second Changzhou People's Hospital Affiliated to Nanjing Medical University, No. 29 XingLong Lane, Tian-Ning District, Changzhou, Jiangsu Province, China, 213003

Tel +8651988104931 Ext. 3077

Fax +8651988119163

Email oncol@medmail.com.cn

\begin{abstract}
Tumor lysis syndrome (TLS), a result of rapid cell lysis following tumor therapy, is a well recognized complication during the treatment of rapidly growing tumors. TLS rarely occurs in solid tumors. We present a case report of TLS in a patient with primary retroperitoneal soft tissue sarcoma. TLS occurred in the patient after four days' combinational chemotherapy with cisplatin, adriamycin, and dacarbazine. These drugs were selected on the basis of an ex vivo ATP-based tumor sensitivity assay. TLS was properly controlled in the patient with concomitant remission of the sarcoma. Therefore, precautions should be taken to avoid this potentially fatal complication during treatment of solid tumors, especially with tumors highly sensitive to drugs.
\end{abstract}

Keywords: tumor lysis syndrome, retroperitoneal soft tissue sarcoma, ATP-based tumor sensitivity assay (ATP-TCA)

\section{Introduction}

Treatment of retroperitoneal soft tissue sarcomas is difficult. Adequate tumor-free margins are not easily obtained and locoregional recurrence is common following surgery for retroperitoneal soft tissue sarcomas. To improve locoregional control, various experts advocate extensive surgery, or suggest the adjuvant use of chemotherapy. Tumor lysis syndrome (TLS), which results from the rapid malignant cell lysis, is a well recognized complication during treatment of rapidly growing tumors. Consequences of rapid cellular destruction include hyperuricemia, hyperkalemia, and hypocalcemia. These abnormalities may lead to acute renal failure. In this case, we describe a rare case of TLS in a man with primary retroperitoneal soft tissue sarcoma after chemotherapy by ex vivo ATP-based tumor chemosensitivity assay (ATP-TCA). ATP-TCA is an effective and easy-to-use methodology to selectively fresh isolated tumor cells. A major advantage over previous approaches is that the ATP-TCA respects the interaction sarcomas and the surrounding stromal cells. Using this test system, we were able to show that retroperitoneal soft tissue sarcomas display remarkable chemosensitivity to different anticancer agents.

\section{Case report}

A 44-year-old man had one-month history of progressively worsening abdominal distention and pain, pantothenic acid, nausea, $5 \mathrm{~kg}$ weight loss. On physical examination, the patient had abdominal eminence and a firm, border-less mass was palpable in central abdominal. Abdominal magnetic resonance imaging (MRI) revealed an occupancy mass of $21 \times 18 \mathrm{~cm}$ in left posterolateral peritoneum, the left kidney 
and lumbar muscle invasion, obstruction of left ureter, and hydronephrosis (Figure 1A). The left renal artery and renal veins were all compressed and the inferior vena cave showed thrombosis (Figure 1C). Laboratory testing revealed that the patient had serum lactate dehydrogenase (LDH) of $1365.4 \mathrm{U} / \mathrm{L}$ (normal range: 50-240 U/L), serum urea nitrogen of $2.8 \mathrm{mmol} / \mathrm{L}$ (normal range: 1.7 to $8.2 \mathrm{mmol} /$ $\mathrm{L}$ ), serum creatinine of $74.3 \mu \mathrm{mol} / \mathrm{L}$ (normal range: 0-124.0 $\mu \mathrm{mol} / \mathrm{L}$ ), serum uric acid of $331.0 \mu \mathrm{mol} / \mathrm{L}$ (normal range: $0-419.0 \mu \mathrm{mol} / \mathrm{L})$, serum calcium of $2.35 \mathrm{mmol} / \mathrm{L}$ (normal range: $2.0-2.6 \mathrm{mmol} / \mathrm{L}$ ), serum phosphate of $1.1 \mathrm{mmol} / \mathrm{L}$ (normal range: $0.8-1.5 \mathrm{mmol} / \mathrm{L}$ ), serum alphafetoprotein (AFP) of $6756 \mu \mathrm{g} / \mathrm{L}$ (normal range: 0-7 $\mu \mathrm{g} / \mathrm{L}$ ), serum neuron-specific enolase (NSE) of $40.37 \mu \mathrm{g} / \mathrm{L}$ (normal range: $0-15.2 \mu \mathrm{g} / \mathrm{L}$ ). Abdominal tumor biopsy samples were obtained using real-time B ultrasound-guided fine needle (16G). The biopsy specimens confirmed a histological diagnosis of primary retroperitoneal soft tissue sarcoma with hematoxylin and eosin staining (Figures 2A, 2B).
Immunohistochemistry demonstrated a positive staining for vimentin (VIM) (Figure 2C), epithelial membrane antigen (EMA) (Figure 2D) and platelet endothelial cell adhesion molecule-1 (PECAM-1). The tumor sections had negative stainings for cytokeratin 7, synaptophysin, chromogranin A, CD57 and cytokeratin 20 (data not shown). The ex vivo biopsy specimens were detected by ATP-TCA testing (ATP-TCA, DCS Innovative Diagnostic Systems ${ }^{\circledR}$, Hamburg, Germany). ${ }^{1}$ It revealed the strong sensitivity of cisplatin (DDP), adriamycin (ADM) and dacarbazine (DTIC), the resistances of paclitaxe, vindesine, bleomycin, ifosfamide, and actinomycin D. The patient was given a combined chemotherapy regimen with DDP $\left(30 \mathrm{mg} / \mathrm{m}^{2}\right.$ intravenously on days 1 through 4), ADM (30 mg/m² intravenously on days 1,3$)$, and DTIC (400 mg/m² intravenously on days 1 through 3 ) on the basis of the ATP-TCA in a three-week cycle. Three days after initiating chemotherapy, the patient felt weary seriously but drowsiness with normal urinary production. Four days after initiating chemotherapy, the patient

A
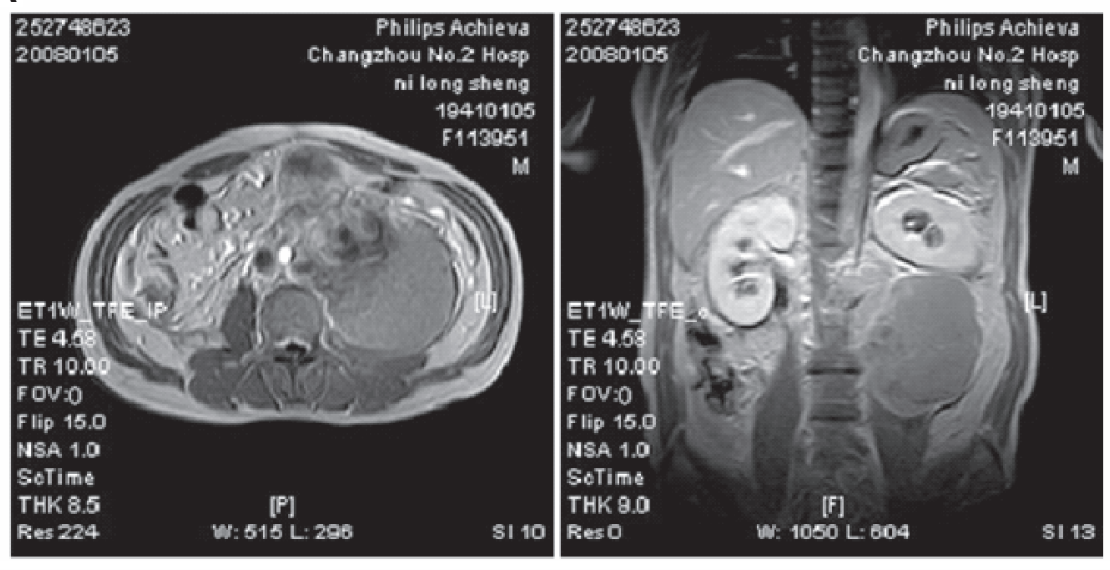

B
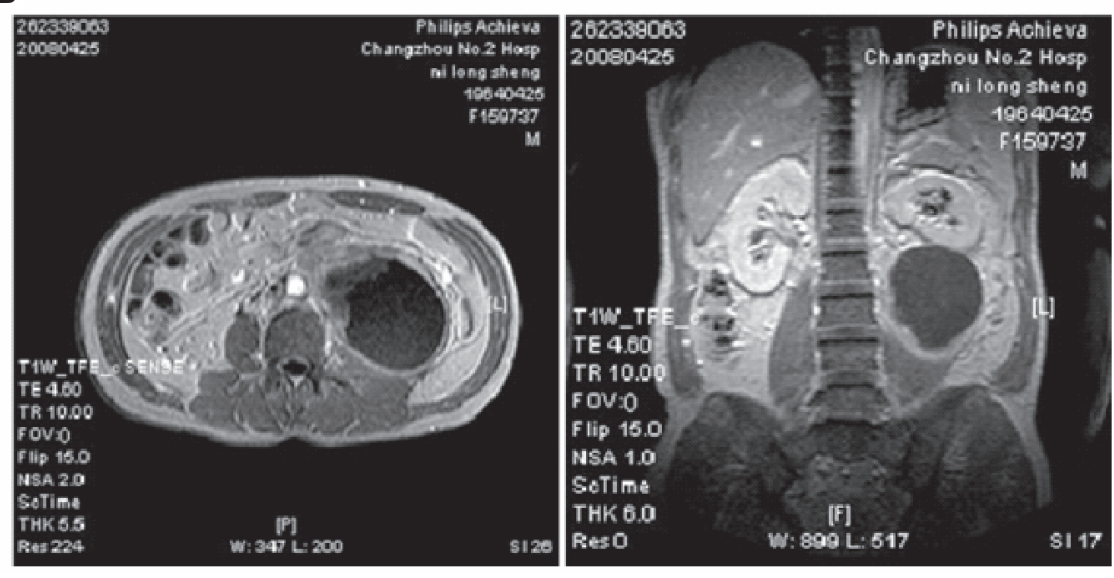

Figure I Magnetic resonance imaging (MRI) shows a bulky abdominal neoplasm at the left posterolateral position of abdomen with compression of kidney, renal artery and renal veins on the left side (A). MRI after 3 cycles of chemotherapy reveals complete remission of the tumor with cystic degeneration and necrosis of the tumor mass (B). 

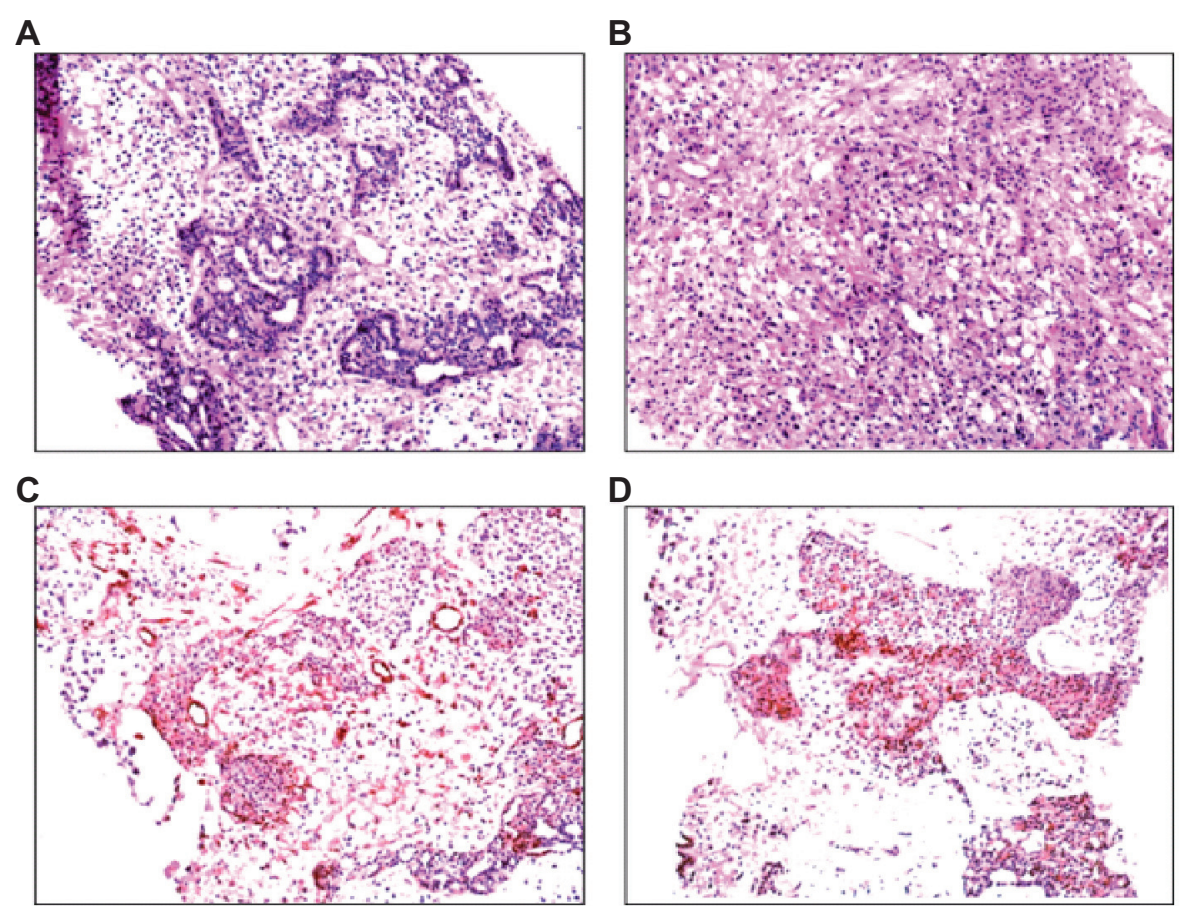

Figure 2 Histological analysis of the biopsy tumor specimen by .hematoxylin and eosin staining of the tumor $(\mathbf{A}, X 100 ; \mathbf{B}, X 400)$ indicates diagnosis of primary retroperitoneal soft tissue sarcoma. The tumor samples were positive in immunohistochemical analysis for vimentin (C) and epithelial membrane antigen (D).

felt chest tightness, palpitations, dyspnea, and oliguria. Laboratory testing revealed evidence of acute renal failure as indicated by serum LDH level of $1565.4 \mathrm{U} / \mathrm{L}$ (normal range: 50-240 U/L), serum urea nitrogen of $7.2 \mathrm{mmol} / \mathrm{L}$ (normal range: $1.7-8.2 \mathrm{mmol} / \mathrm{L}$ ), serum creatinine of $143.0 \mu \mathrm{mol} / \mathrm{L}$ (normal range: $0-124.0 \mu \mathrm{mol} / \mathrm{L}$ ), serum uric acid of $584.0 \mu \mathrm{mol} / \mathrm{L}$ (normal range: $0-419.0 \mu \mathrm{mol} / \mathrm{L}$ ), serum calcium of $1.7 \mathrm{mmol} / \mathrm{L}$ (normal range: $2.0-2.6 \mathrm{mmol} / \mathrm{L}$ ), and serum phosphate of $2.3 \mathrm{mmol} / \mathrm{L}$ (normal range: $0.8-1.5 \mathrm{mmol} / \mathrm{L}$ ). An ultrasonographic examination showed no evidence of extrarenal obstruction or renal vein thrombosis. A chemotherapy-induced tumor lysis syndrome (TLS) generated and DDP was immediately discontinued. Metabolic abnormalities were treated successfully using a combination of sodium bicarbonate, furosemide, and allopurinol. The serum levels of urea nitrogen, creatinine, uric acid, calcium, and phosphate were normalized. The blood LDH level decreased to 365.4 U/L on day 12 after the treatment. The second and the third chemotherapy cycles were continued until three weeks after initiation of chemotherapy, accompanying with allopurinol, diuretic, alkaline urine. The therapeutic effects of the patient were evaluated at the 30th day following the third chemotherapy cycle. The patient's abdominal distention and pain were relieved. The abdominal mass was impalpable on physical examination. The abdominal MRI revealed the tumor was partial remission with cystic degeneration and the mass was necrosis (Figure 1B). Laboratory testing revealed a serum LDH level of $143.0 \mathrm{U} / \mathrm{L}$, serum AFP of $510.0 \mu \mathrm{g} / \mathrm{L}$ and NSE of $14.7 \mu \mathrm{g} / \mathrm{L}$.

\section{Discussion}

TLS is observed most frequently in patients with hematologic malignancies such as acute lymphoblastic leukemia (ALL) and Burkitt's lymphoma after the initiation of chemotherapy, although it may also occur in other malignancies, both hematologic and solid tumors. For the past few years, a variety of solid tumors have been reported to be associated with this syndrome, ${ }^{2}$ including tumors in the brain, lung, ovary, stomach, and skin..$^{3-5}$ Our work presented a case of TLS in a patient with primary retroperitoneal soft tissue sarcoma that occurred after four days' chemotherapy with DDP, ADM, and DTIC. DDP can lead to clinical and laboratory evidence of acute renal failure, but mainly damaged renal epithelial, not typical of the TLS metabolic disorder and slow restoration. This is a typical case of metabolic disorders initiating chemotherapy and no similar syndrome in further chemotherapy. We speculate DDP lead to acute renal failure can be ruled out.

Certain intrinsic tumor-related factors have been associated with an increased risk for the development of TLS, including high tumor cell proliferation rate, large tumor burden, tumor chemosensitivity, and increased LDH levels. 
In our patient, several risk factors might predispose TLS. The first risk factor was chemosensitivity for primary retroperitoneal soft tissue sarcoma. Soft tissue sarcomas comprise less than $1 \%$ of all solid malignancies. The role of chemotherapy is not clearly defined and is largely restricted to clinical trials. Only a limited number of agents have proved to be effective in soft tissue sarcomas. Lehnhardt and colleagues also reported sensitivity testing in soft tissue sarcomas. ${ }^{6}$ It is expected that the pre-therapeutic sensitivity testing of cytotoxicity with ATP-TCA would provide the opportunity of improving the success rate of conventional chemotherapy in soft tissue sarcomas, as observed in this study. However, individualized chemotherapy protocol evaluated by ATP-TCA testing should be combined with clinical data in practice. The combination of chemotherapy used in the patient with primary retroperitoneal soft tissue sarcoma was chosen by an ATP-TCA testing system to determine the sensitivity of cultured tumor cells to various anti-tumor drugs. The biopsy specimens were difficult to achieve for inoperableness patient. In this case, abdominal tumor biopsy can be done by cutting a small piece of the tissue specimens on real-time B ultrasound-guided fine needle. The strong sensitivity of DDP, ADM and DTIC by ex vivo ATP-TCA in the patient was confirmed with TLS. The initiating chemotherapy and the abdominal MRI revealed partial remission of the tumor with cystic degeneration and necrosis of the tumor mass. The next risk factor was large tumor burden in the patient. Abdominal MRI revealed a giant occupancy mass of $21 \times 18 \mathrm{~cm}$ in left posterolateral peritoneum, the left kidney, and lumbar muscle invasion. The third risk factor was increased LDH levels. The serum LDH level was markedly increased in the patient, accompanied with a slight increase of serum uric acid level before the initiating chemotherapy. The fourth risk factors was urethral obstruction and kidney blood flow reduction in the patient. Abdominal MRI revealed obstruction of left ureter, hydronephrosis, the left renal artery and renal veins were all compressed, and inferior vena cave was thrombosis. The cellular components from malignant cells rapid lysis with chemotherapy into the blood following significantly reduced, induced, or exacerbated TLS.

In our view, precautions should be taken to avoid this potentially fatal complication when treating solid tumors, especially in drug-sensitive tumors with bulky size and rapid progression. Several pre-existing risk factors such as azotemia, elevated LDH, and hyperuricemia should also be considered for the potential development of TLS. In summary, identifying patients at risk, implementing preventive measures and initiating prompt therapies to treat TLS at early recognition are all essential for avoiding serious complications associated with TLS.

\section{Acknowledgments}

This work was supported by research grants from "the Technology Project of Changzhou Social Development" (CS2008217) to C-JQ.

\section{References}

1. Kurbacher CM, Cree IA. Chemosensitivity testing using microplate adenosine triphosphate-based luminescence measurements. Methods Mol Med. 2005;110:101-120.

2. Drakos P, Bar-Ziv J, Catane R. Tumor lysis syndrome in nonhematologic malignancies. Report of a case and review of the literature. Am J Clin Oncol. 1994;17:502-505.

3. Ajzensztejn D, Hegde VS, Lee SM. Tumor lysis syndrome after treatment with docetaxel for non-small-cell lung cancer. J Clin Oncol. 2006;24:2389-2391.

4. Han HS, Park SR, Kim SY, et al. Tumor lysis syndrome after capecitabine plus cisplatin treatment in advanced gastric cancer. J Clin Oncol. 2008;26:1006-1008.

5. Baeksgaard L, Sorensen JB. Acute tumor lysis syndrome in solid tumors - a case report and review of the literature. Cancer Chemother Pharmacol. 2003;51:187-192.

6. Lehnhardt M, Muehlberger T, Kuhnen C, et al. Feasibility of chemosensitivity testing in soft tissue sarcomas. World J Surg Oncol. 2005;3:20. 